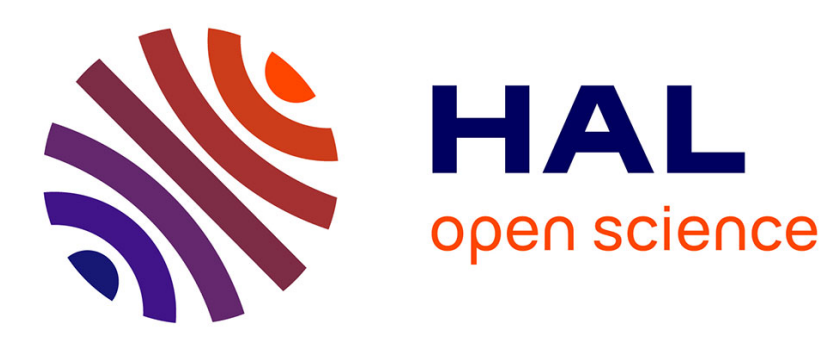

\title{
Mesure de la vitesse de déplacement d'un arc de coupure sur une electrode plane
}

\author{
P. Andanson, A. Lefort, J. Roche
}

\section{To cite this version:}

P. Andanson, A. Lefort, J. Roche. Mesure de la vitesse de déplacement d'un arc de coupure sur une electrode plane. Revue de Physique Appliquée, 1978, 13 (12), pp.873-876. 10.1051/rphysap:019780013012087300 . jpa-00244563

\section{HAL Id: jpa-00244563 https://hal.science/jpa-00244563}

Submitted on 1 Jan 1978

HAL is a multi-disciplinary open access archive for the deposit and dissemination of scientific research documents, whether they are published or not. The documents may come from teaching and research institutions in France or abroad, or from public or private research centers.
L'archive ouverte pluridisciplinaire HAL, est destinée au dépôt et à la diffusion de documents scientifiques de niveau recherche, publiés ou non, émanant des établissements d'enseignement et de recherche français ou étrangers, des laboratoires publics ou privés. 
Classification

Physics Abstracts

52.80

\title{
MESURE DE LA VITESSE DE DÉPLACEMENT D'UN ARC DE COUPURE SUR UNE ÉLECTRODE PLANE
}

\author{
P. ANDANSON, A. LEFORT et J. ROCHE
}

Groupe de Physique des Matériaux, Université de Clermont II, B.P. 45, 63170 Aubière, France

(Reçu le 21 juin, révisé le 13 septembre 1978, accepté le 18 septembre 1978)

\begin{abstract}
Résumé. - Dans cet article les auteurs montrent comment ils ont mesuré la vitesse de déplacement sur une surface plane métallique, d'un pied d'arc électrique parcouru par un courant compris entre 500 et $3000 \mathrm{~A}$. Le dispositif expérimental réalisé permet d'effectuer les mesures nécessaires en temps réel sans perturber le phénomène. Les résultats illustrent le caractère instable du plasma et montrent que le déplacement s'effectue de manière aléatoire.
\end{abstract}

Abstract. - In the present work, the authors explain their way of measuring the speed at which the spot of an electric arc moves along a flat metallic surface, when carrying a current from 500 to $3000 \mathrm{~A}$.

The set-up devised enables the necessary measurements to be made in real time without perturbing the process.

The results illustrate the unsteady character of plasma and show that the motion occurs randomly.

1. Introduction. - Les constructeurs d'appareil de coupure pour des courants d'intensité moyenne, provoquent le déplacement, après l'ouverture du disjoncteur des pieds d'arc vers une partie pouvant subir des dégradations sans compromettre l'état de surface des contacts. Ceci est possible du fait de la grande mobilité des pieds d'arc. Cette mobilité a été étudiée par le passé dans le cas d'arcs au mercure [1, $2,3]$. Mais la plupart du temps les conditions expérimentales perturbent le phénomène par la présence de capteurs situés à proximité immédiate de la surface des électrodes. Nous avons utilisé un système de visées optiques associé à un dispositif électronique qui permet de mesurer les intervalles de temps nécessaires à l'obtention des vitesses. Les résultats obtenus concernent le cuivre et l'argent pour les taches cathodiques et anodiques.

2. Le montage expérimental. - 2.1 LE SUPPORT DE DÉPLACEMENT. - Ces expériences ont eu lieu à la station d'essai des usines Landis et Gyr de Montluçon. La création de l'arc et l'enregistrement des paramètres électriques courant et tension sont les mêmes que dans le cas de notre étude concernant l'érosion des contacts [4].

Nous décrivons brièvement le déroulement d'une expérience. Le disjoncteur sur lequel porte l'étude est disposé fermé dans le circuit d'essai, en série avec un court-circuiteur ouvert et les impédances néces- saires pour obtenir les valeurs des déphasages couranttension désirées ainsi que l'amplitude de l'intensité. A un passage à zéro de la tension sinusoïdale délivrée par le secondaire du transformateur de $1600 \mathrm{kVA}$ le court-circuiteur se ferme. Après un temps de réponse variant entre $0,001 \mathrm{~s}$ et $0,003 \mathrm{~s}$ selon les appareils, le disjoncteur en essai s'ouvre et il apparaît un arc électrique qui va s'éteindre lors du passage à zéro suivant de la tension. Comme nous travaillons avec un $\cos \varphi$ égal à un, les réamorçages sont extrêmement rares et la durée de l'arc étudié se situe donc entre $0,009 \mathrm{~s}$ et $0,007 \mathrm{~s}$.

Toutefois l'appareil de coupure sur lequel ont porté les mesures n'est pas le même pour des raisons de performance et de géométrie. En particulier, le temps de retard à l'ouverture est dans ce cas de 0,002 s. Les modifications effectuées pour faciliter les mesures nous ont conduits à supprimer le système de déclenchement différentiel ainsi que les tôles déions. Le contact fixe sur lequel le pied d'arc se déplace a lui aussi été modifié. Dans le cas d'expériences relatives à un support en argent nous avons soudé au four H.F. sur le porte-contact, une bande constituée par ce métal et ayant une épaisseur de $0,001 \mathrm{~m}$, une largeur de $0,005 \mathrm{~m}$ et une longueur de $0,030 \mathrm{~m}$. Pour les mesures relatives au cuivre c'est le porte-contact constitué par ce métal qui a servi de support au pied d'arc.

Après l'ouverture du disjoncteur, comme le montre 
la figure 1, l'une des extrémités de l'arc se déplace sur le contact fixe. C'est cette vitesse de déplacement que nous avons mesurée.

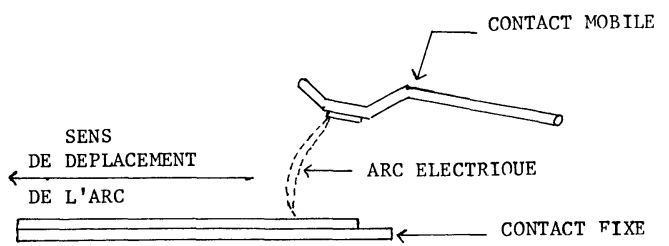

Fig. 1. - Schéma du développement de l'arc entre les contacts.

[Diagram of arc extension between contacts.]

2.2 LE SYSTÈME DE VISÉES OPTIQUES. - Le capteur utilisé est constitué par un ensemble de 7 fibres optiques recevant la lumière émise par le pied d'arc dans une direction perpendiculaire à la fois au déplacement de celui-ci et à la normale à la surface de la tache en mouvement (Fig. 1); cette direction de visée est donc parallèle au plan matérialisé par la surface du contact fixe. Les fibres sont montées dans un support dont nous donnons une vue en coupe (Fig. 2). Pour avoir une boonne précision sur l'instant de passage du centre du pied d'arc (partie la plus lumineuse de celui-ci), nous avons collimaté la fenêtre de visée par des trous de diamètre $0,0003 \mathrm{~m}$ percés dans la masse du support en laiton et dans la pièce isolante en bakélite.

Le guide de lumière est constitué par des fibres de $45 \times 10^{-6} \mathrm{~m}$ de diamètre, groupées en faisceau de

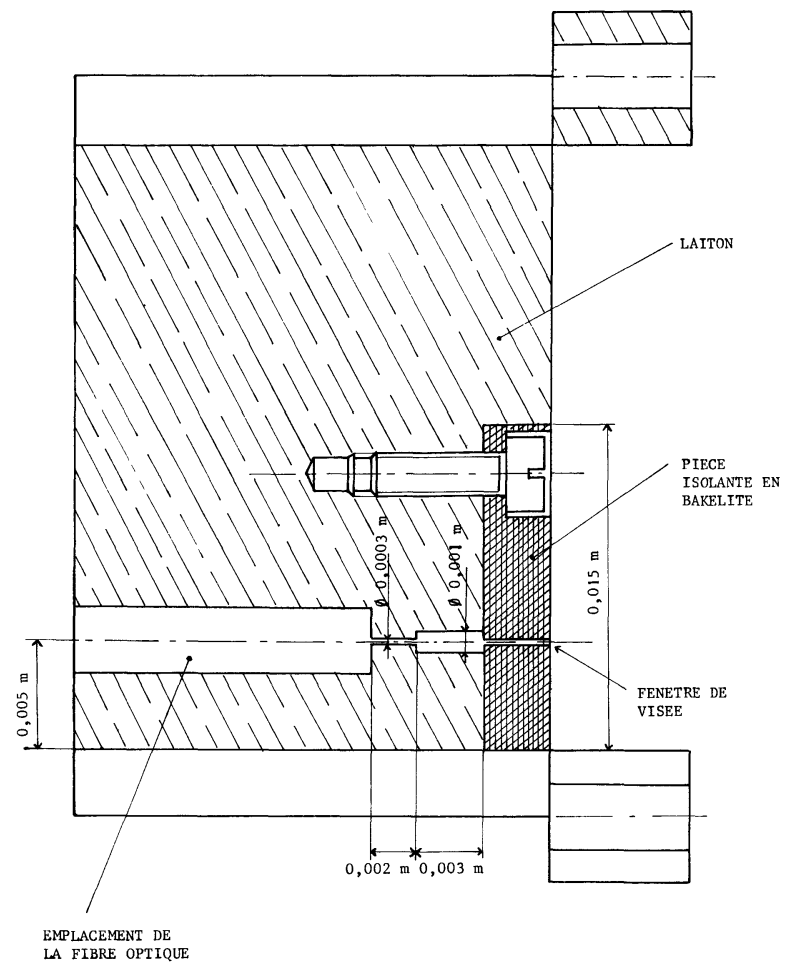

FIG. 2. - Montage du capteur de mesure.

[Setting of measure detector.]
$0,001 \mathrm{~m}$ de diamètre. Le rendement lumineux pour une longueur de $3 \mathrm{~m}$ est de 0,4 , l'ouverture numérique vaut $66^{\circ}$ et la réponse spectrale est linéaire de $460 \times 10^{-9} \mathrm{~m}$ à $1300 \times 10^{-9} \mathrm{~m}$. La protection du faisceau est assurée par un gainage plastique. Les embouts ont été équipés de façon à pouvoir d'un côté s'adapter sur le support en laiton et de l'autre côté se raccorder à un phototransistor par une prise de type BNC.

2.3 Le MONTAGE ÉleCtroniQue. - Nous donnons (Fig. 3) le schéma synoptique de la partie électronique du montage. Nous allons décrire très rapidement son fonctionnement.

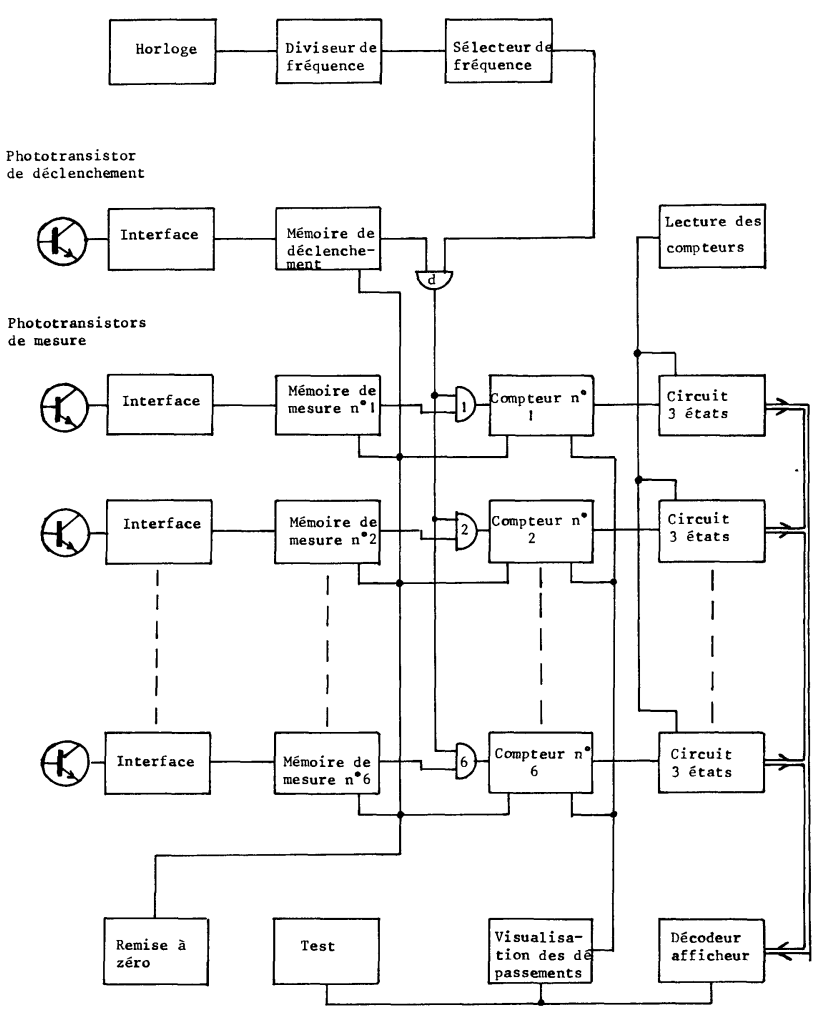

Fig. 3. - Schéma synoptique du montage électronique.

[Synopsis of electronic setting.]

Le but de ce montage est de mesurer les temps mis par le pied d'arc pour atteindre les positions relatives aux différents points de visée.

L'horloge délivre des impulsions carrées de $1 \mathrm{MHz}$. Grâce au diviseur de fréquence associé nous pouvons obtenir la moitié, le quart et le huitième de cette valeur. La gamme choisie dépend de la vitesse du pied d'arc sur son support.

Les trous de visée, au nombre de 7, sont disposés le long du contact fixe à une distance de $0,0045 \mathrm{~m}$ les uns des autres, le premier se situe au niveau du point de contact des électrodes. Lorsque le disjoncteur, sous l'effet du passage du courant électrique s'ouvre, la fibre associée à ce premier trou est éclairée et débloque le premier phototransistor du montage 
que nous appelons phototransistor de déclenchement. La porte " $\mathrm{d}$ " reçoit un signal de la mémoire de déclenchement et permet le passage des signaux délivrés par l'horloge. Les compteurs 1 à 6 , préalablement remis à zéro, enregistrent les impulsions délivrées par l'horloge.

Lorsque le pied d'arc passe devant la fenêtre de visée de la fibre optique suivante, la porte numéro 1 reçoit un signal du phototransistor correspondant et bloque le compteur numéro 1 . Les compteurs suivants sont bloqués lorsque le pied d'arc passe devant les fibres optiques correspondantes. Après lecture des mémoires des six compteurs, et connaissant la fréquence délivrée par l'horloge, nous avons de façon simple la vitesse moyenne du pied d'arc entre deux points séparés de $0,0045 \mathrm{~m}$, et aussi entre deux points multiples (jusqu'au facteur 6) de cette distance.

3. Les résultats expérimentaux. - Nous rappelons que l'arc électrique étudié n'existe que pendant une demi-alternance d'un courant sinusoïdal de fréquence $50 \mathrm{~Hz}$. Les valeurs de l'intensité que nous donnerons dans la suite de cet article sont les valeurs moyennes calculées sur le temps de déplacement de l'arc le long du contact fixe. L'instant de fin de mesure étant proche du maximum de courant.

L'expérience nous a tout d'abord montré que les compteurs portant les numéros 5 et 6 donnent les valeurs inférieures aux compteurs 3 et 4 pour les faibles valeurs de l'intensité du courant. Ceci nous conduit à émettre l'hypothèse selon laquelle l'arc électrique se scinderait en deux parties comme nous l'avons schématisé sur la figure 4. Il faut noter que ce phénomène a lieu aussi bien lorsque le contact fixe joue le rôle d'anode que celui de cathode, et cesse simultanément dans ces deux cas pour une intensité donnée.

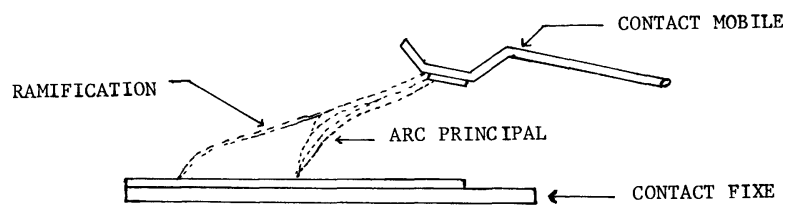

FIG. 4. - Cas de l'arc avec une ramification.

[Case of a branching arc.]

On peut émettre les hypothèses suivantes :

- La ramification existe dès que l'arc apparaît mais elle n'est pas suffisamment lumineuse lorsqu'elle passe devant les fibres 3 et 4 pour permettre l'arrêt des compteurs 3 et 4 .

- Sous l'effet des irrégularités de la surface de l'électrode utilisée, le champ électrique prend localement des valeurs importantes qui permettent l'amorçage d'un deuxième arc au niveau des fibres 5 et 6 .
- Le pied d'arc reste accroché sur le contact mobile en un point qui est pratiquement fixe. L'érosion extrêmement importante provoque l'apparition de grains de métal fondu qui projetés hors de la colonne d'arc favorisent l'amorçage qui donnera naissance à la ramification.

Une étude par caméra ultra-rapide devrait permettre de préciser l'une de ces trois hypothèses.

Dans le cas d'un support en cuivre ce phénomène cesse lorsque l'intensité du courant atteint des valeurs supérieures à $1800 \mathrm{~A}$. Pour le support en argent, bien que nous ayons fait une étude jusqu'à $2600 \mathrm{~A}$, nous avons toujours l'apparition d'un deuxième arc au niveau de la dernière fibre de visée. D'après les observations faites sur nos résultats, en particulier pour les contacts en argent où ce phénomène est toujours présent, les deux pieds d'arc sont séparés par une distance variant de $0,014 \mathrm{~m}$ (pour $600 \mathrm{~A}$ ) à $0,009 \mathrm{~m}$ (pour $2600 \mathrm{~A}$ ). Dans le cas du cuivre nous retrouvons la même valeur que précédemment pour $600 \mathrm{~A}$.

Ce deuxième arc constitue en réalité une ramification de l'arc principal. Nous l'avons démontré en plaçant devant la fibre, reliée au compteur numéro 6 , un filtre qui permet d'atténuer l'intensité lumineuse. Dans ce cas le compteur numéro 6 rend seulement compte du passage de l'arc principal qui est beaucoup plus lumineux que la ramification.
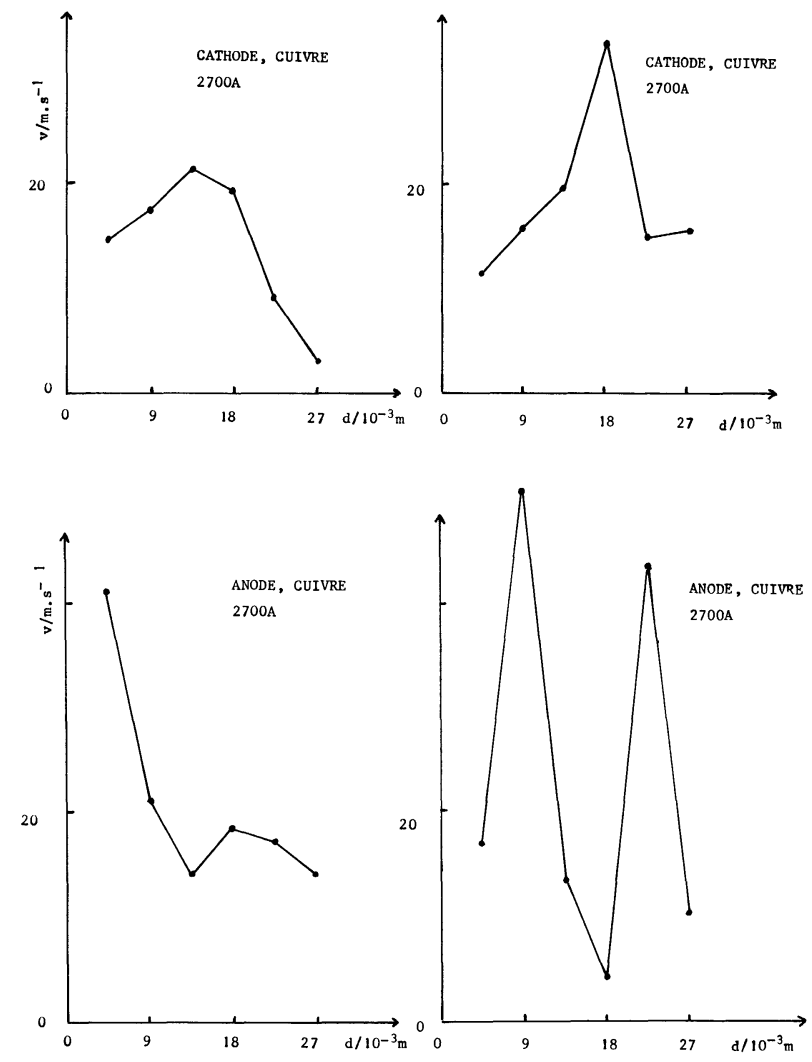

FIG. 5. - Exemples de vitesses obtenues dans le cas d'un support en cuivre.

[Examples of speedssobtained in case of a copper contact.] 
Nous allons maintenant voir le résultat de la mesure de la vitesse des pieds d'arc.

En effectuant le calcul de la vitesse du pied d'arc, en utilisant les résultats donnés par deux compteurs successifs, nous avons une indication sur les variations de cette vitesse au cours du temps. Il revient pratiquement au même de représenter ces vitesses en fonction des distances parcourues. La précision relative des résultats obtenus est meilleure que $1 \%$. Des exemples d'une telle représentation sont donnés par les figures 5 et 6 . Nous avons joint les points expérimentaux par des segments de droite afin de mieux visualiser le phénomène. Ces résultats montrent le caractère aléatoire de la vitesse de déplacement du pied d'arc, et ce aussi bien à l'anode qu'à la cathode pour les deux matériaux utilisés. Ceci confirme les observations faites par G. Burkhard [5].

Enfin nous donnons les vitesses moyennes du pied d'arc calculées à partir des données du compteur numéro 6 lorsque l'arc est unique, et à partir de celles données par les compteurs 4 et 5 dans les autres cas.

Ces résultats sont donnés par les figures 7 et 8 . Les barres d'erreur traduisent le caractère aléatoire du phénomène mesuré.
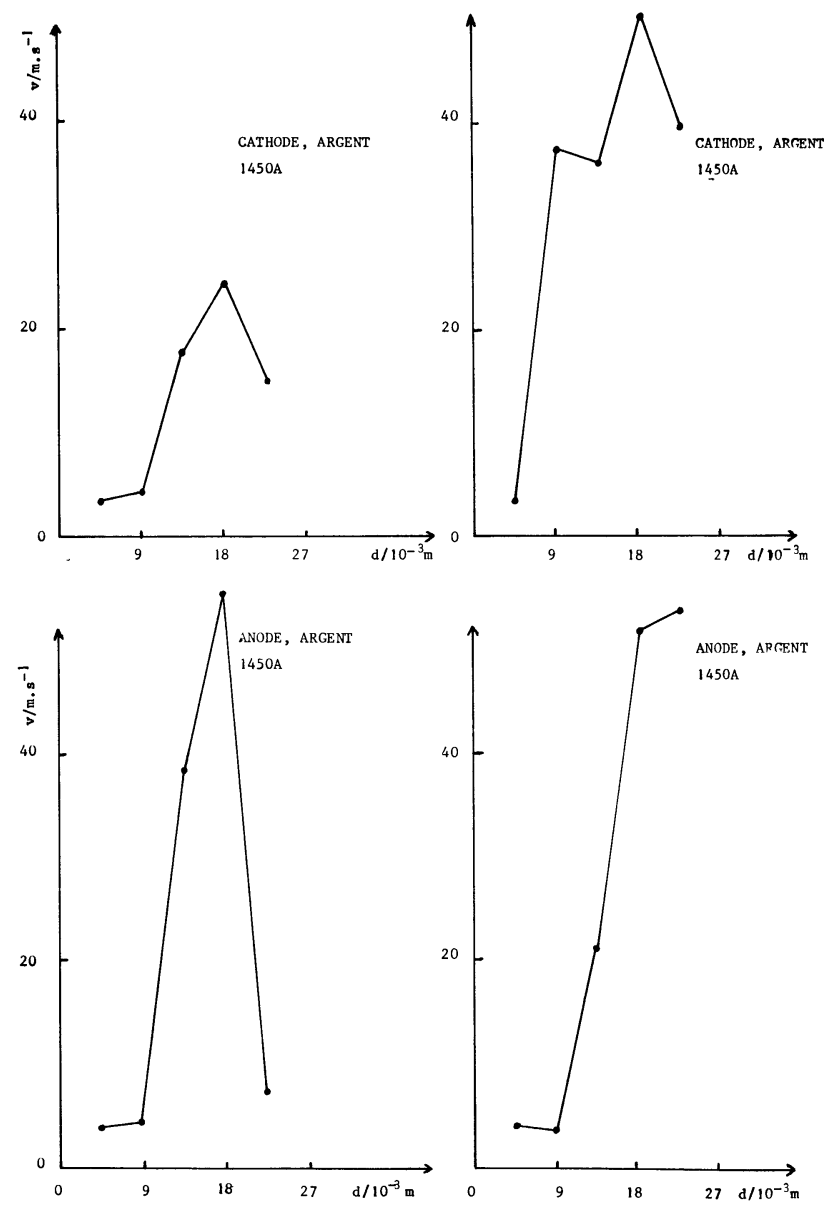

Fig. 6. - Exemples de vitesses obtenues dans le cas d'un support en argent.

[Examples of speeds obtained in case of a silver contact.]

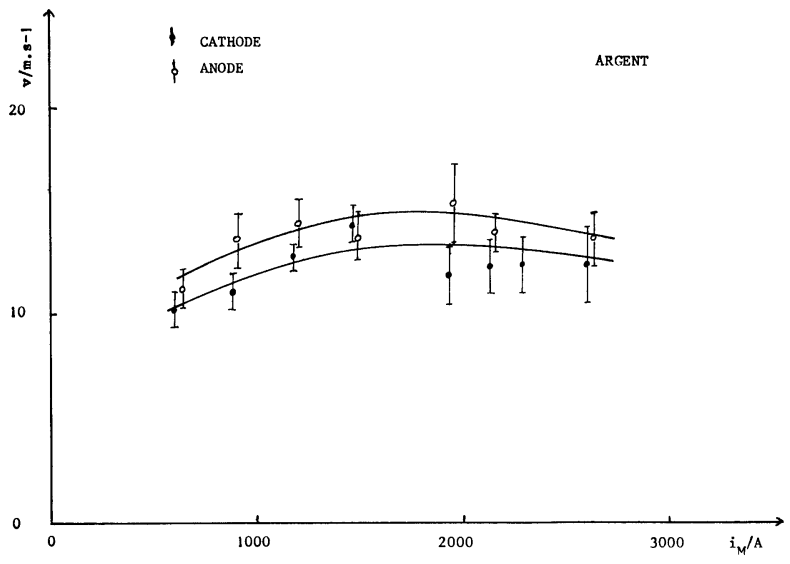

FIG. 7. - Les vitesses moyennes du pied d'arc sur des contacts en argent.

[Mean speeds of are spot on silver contacts.]

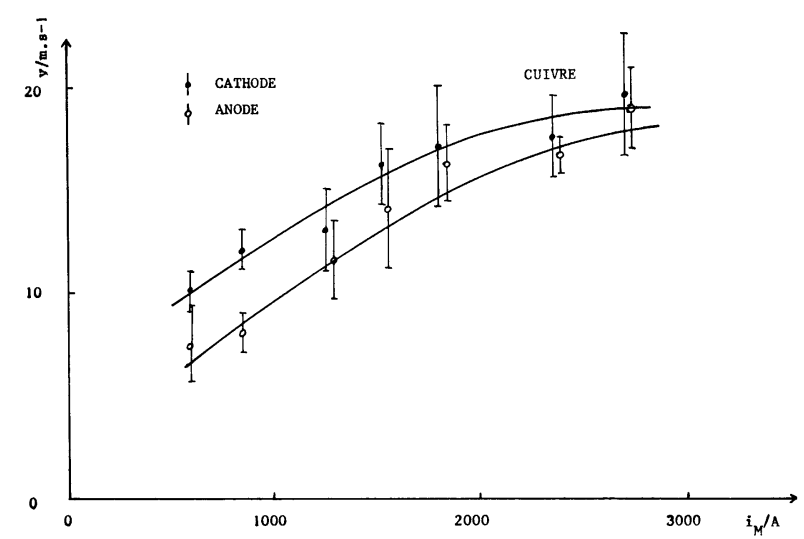

FIG. 8. - Les vitesses moyennes du pied d'arc sur des contacts en cuivre.

[Mean speeds of are spot on copper contacts.]

4. Conclusion. - Si nous nous intéressons aux valeurs des vitesses moyennes nous remarquons que les supports sur lesquels se déplace le pied d'arc conduisent à des valeurs différentes. Dans le cas de l'argent les vitesses varient autour d'une valeur que l'on peut situer entre $11 \mathrm{~m} . \mathrm{s}^{-1}$ et $15 \mathrm{~m} . \mathrm{s}^{-1}$, et la tache anodique est ici la plus rapide.

Pour un support en cuivre, les vitesses augmentent avec l'intensité du courant. Les différences entre les phénomènes à l'anode et à la cathode sont plus marquées que dans le cas précédent, mais se réduisent lorsque l'intensité du courant atteint des valeurs voisines de $2700 \mathrm{~A}$. Et dans ce cas les vitesses de déplacement de la tache cathodique sont plus grandes que celles de la tache anodique.

Enfin, pour conclure nous remarquerons que les pieds d'arc se déplacent plus rapidement sur un support en cuivre que sur un support en argent.

\section{Bibliographie}

[1] Callagher, C. J. et Cobine, J. D., Phys. Rev. 71 (1974) 481.

[2] Smith, C. G., Phys. Rev. 73 (1948) 543.

[3] Robson, A. E. et Engel, A. V., Phys. Rev. 93 (1954) 1121.

[4] Andanson, P., Bouculat, M., Cheminat, B., Lefort, A. et Roche, J., Revue Phys. Appl. 12 (1977) 999.

[5] BURKHARD, G., Elektric 24 (1970) 1. 